\title{
Case Report \\ Partners in Crime in the Setting of Recurring Cardiac Arrest
}

\author{
Lida P. Papavasileiou, Giovanni B. Forleo, Luca Santini, Eugenio Martuscelli, \\ and Francesco Romeo
}

Cardiology Department, Tor Vergata University Hospital of Rome, Viale Oxford 81, 00133 Rome, Italy

Correspondence should be addressed to Lida P. Papavasileiou, lidapieretta@hotmail.com

Received 19 October 2010; Revised 28 November 2010; Accepted 15 January 2011

Academic Editor: Vladimír Džavík

Copyright (C 2011 Lida P. Papavasileiou et al. This is an open access article distributed under the Creative Commons Attribution License, which permits unrestricted use, distribution, and reproduction in any medium, provided the original work is properly cited.

No previous reports are available about the potential dramatic effects resulting from the combination of acquired long QT interval not associated to bradycardia and myocardial ischemia. We report the case of a man that during acute necrotic pancreatitis presented QT interval prolongation without bradycardia, TdP, and two episodes of cardiac arrest. A coronary angiogram revealed a subocclusive stenosis of left anterior descending coronary artery, treated with a percutaneous coronary intervention. After myocardial revascularization, even in presence of long QT interval, no arrhythmic events occurred suggesting the key role of myocardial ischemia in triggering TdP in acquired long QT even without bradycardia. ECG performed six months later, after complete recovery from pancreatitis, showed a normal QT interval.

\section{Introduction}

Acquired long QT may be caused by many clinical conditions and is associated with increased risk, during bradycardia, of torsades de pointes (TdP). Myocardial ischemia is also demonstrated to provoke QT interval prolongation. Nevertheless, recent studies have shown that a long QT interval is not sufficient to provoke TdP [1]. No previous reports are available about the potential and dramatic effects resulting from the combination of acquired long QT interval not associated to bradycardia and myocardial ischemia. We report the unique case of a 70-year-old man that during acute necrotic pancreatitis presented QT interval prolongation without bradycardia, TdP, and two episodes of cardiac arrest. A coronary angiogram revealed a critical-subocclusive stenosis of left anterior descending coronary artery, treated with a percutaneous coronary intervention.

\section{Case Report}

A 70-year-old man with hypertension and diabetes but without previous cardiac history, was admitted to general surgery for nausea, abdominal pain, diarrhea, short breath, and weight loss. A CT scan revealed acute biliary pancreatitis so treatment with octretide, piperacillin, and parenteral nutrition was initiated. ECG showed sinus tachycardia, QTc $442 \mathrm{~ms}$ (Figure 1(a)). Clinical status of patient deteriorated during hospital stay as massive necrotic pancreatitis occurred. Serial ECG's showed an increasing QTc interval and episodes of TdP (Figure 1(b)). Fifteen days later, he had a cardiac arrest due to ventricular fibrillation. After resuscitation, he was transferred to our emergency unit. ECG showed sinus rhythm (68 bpm), QTc 683 ms (Figure 2(a)). Echocardioghaphy showed normal left ventricular function without abnormal findings regarding valves and contractility. Cardiac enzymes were negative. Potassium level was 3,2 mEq/L and magnesium 1,9 mEq/L. Infusion of electrolytes corrected disbalance. Continuous ECG revealed frequent episodes of TdP (Figure 2(b)) so antiarrhythmic treatment was considered. Initially bolus of lidocaine and then amiodarone where used, with only temporaneous benefit so antiarrhythmic treatment was abandoned. Four days later, in presence of long QT interval but without bradycardia, a second cardiac arrest occurred due to TdP that degenerated to ventricular fibrillation (Figures 3(a) and 3(b)). After resuscitation, cardiac enzymes were negative, electrolytes were normal, and ECG revealed sinus rhythm with QT interval prolongation without signs of acute ischemia. In order to exclude coronary artery disease, a coronary angiogram was performed and a subocclusive obstruction of left anterior descending artery 


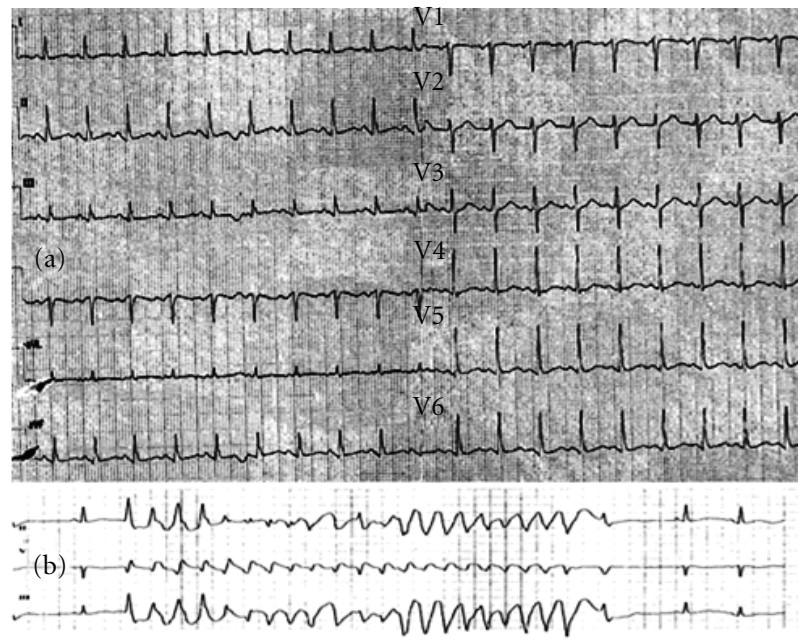

FIgUre 1: (a) Baseline ECG: sinus tachycardia (119bpm), QTc $441 \mathrm{~ms}$. (b) self-terminated episode of TdP.

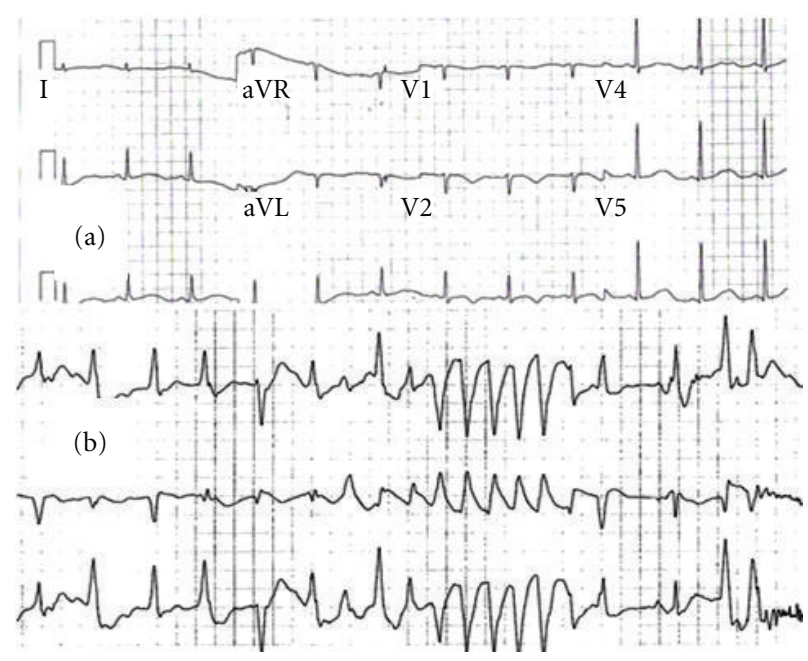

FIGURE 2: (a) ECG after cardiac arrest: Sinus rhythm (68 bpm), QTc $683 \mathrm{~ms}$. (b) Self-terminated episodes of TdP.

was treated with percutaneous coronary intervention and direct stenting (Figures 4(a) and 4(b)). After successful revascularization, QTc interval remained prolonged (Figure 5). A dual chamber defibrillator was implanted. Thereafter, even if patient had not recovered for pancreatitis, no arrhythmic events occurred although QT interval remained prolonged. Complete recovery was achieved only six months later and an ECG performed showed normal sinus rhythm (73 bpm) with normal QT interval while patient was assuming b-blockers (Figure 6).

\section{Discussion}

We report the unusual and potentially lethal manifestation of the combination of acute necrotic pancreatitis and acquired long QT not associated to bradycardia probably triggered by myocardial ischemia.

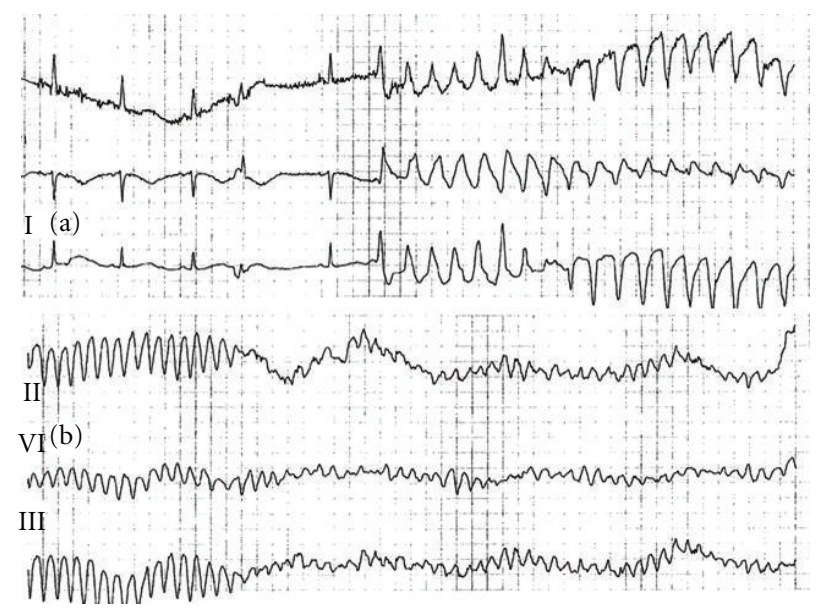

Figure 3: $(a, b)$ TdP degenerating to ventricular fibrillation.

Acute pancreatitis and chronic liver diseases are often associated with electrocardiographic abnormalities [1]. There are also reported QT interval prolongation and QT interval dispersion associated with increased mortality $[2,3]$.

In addition, early transmural ischemia consistently prolongs the Bazett's QTc interval [4]. But long QT interval alone is not sufficient to provoke TdP [5]. Indeed, acquired long QT is associated with increased risk of TdP and only rarely associated with sustained ventricular arrhythmias. Reports mainly attribute TdP to drug-induced QT prolongation, and only rarely TdPs have been associated with acute pancreatitis [1], acute myocardial infarction, or after successful PCI $[5,6]$.

In our case, acute pancreatitis created ventricular vulnerability, by means of QT prolongation, as previously reported [1-3], and we presume that myocardial ischemia ulteriorly prolonged QT interval and triggered TdP and ventricular fibrillation. Classical signs of myocardial ischemia were not present or might have been partially masked by acute pancreatitis. Nevertheless, we feel safe to exclude myocardial infarction considering the negative myocardial enzymes.

Thus, in our belief, the recurring TdP and VF associated with acquired long QT (probably due to acute pancreatitis) were the only clinical manifestation of critical-subocclusive LAD stenosis causing transient myocardial electrical instability. Both hemodynamic events, acute pancreatitis and LAD stenosis, were responsible for $\mathrm{TdPs}$ and ventricular fibrillation, with a critical role of myocardial ischemia as a trigger of ventricular arrhythmias and TdP. In fact, they were present for only a few hours after successful PCI although QT interval prolongation persisted. Decision to implant a defibrillator was based on the inability to predict new events in a patient, in which the combination of acute and recurrent pancreatitis, QT interval prolongation, and critical coronary disease created a potentially lethal effect. In addition, the patient needed to start anti-ischemic treatment with bblockers that may induce bradycardia and potentially trigger ventricular arrhythmias in QT interval prolongation.

Long QT when associated with myocardial ischemia might lead to potentially lethal ventricular arrhythmias. 


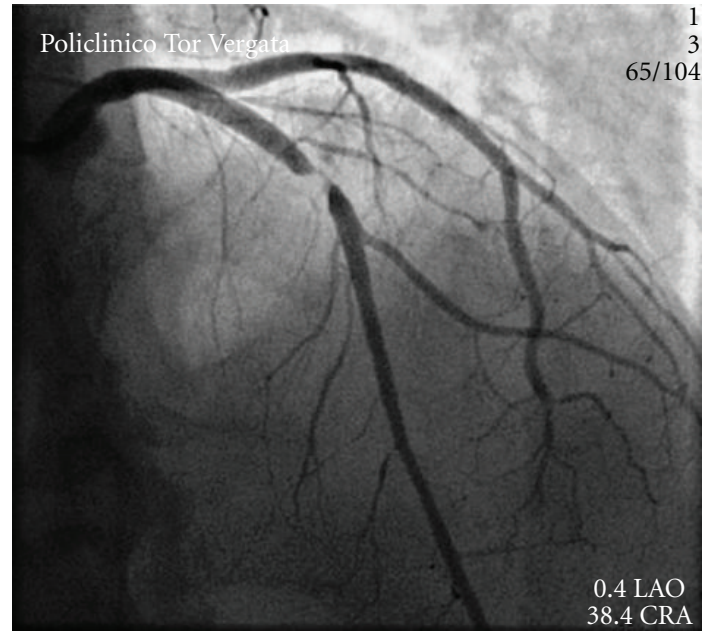

(a)

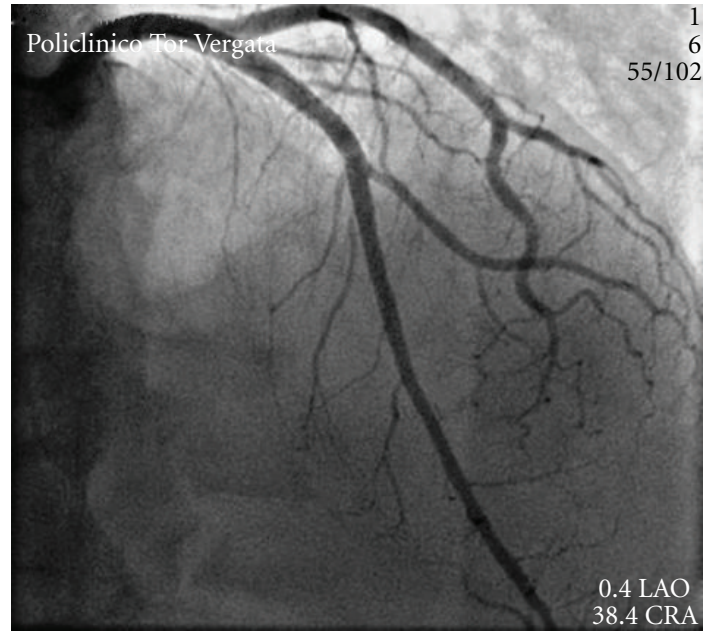

(b)

FIgURE 4: (a) Baseline coronary angiogram (LAO 40, CRA $38^{\circ}$ ) and (b) coronary angiogram after successful percutaneous intervention and direct stenting of the $\mathrm{LAD}\left(\mathrm{LAO} 40^{\circ}\right.$, CRA $\left.38^{\circ}\right)$.

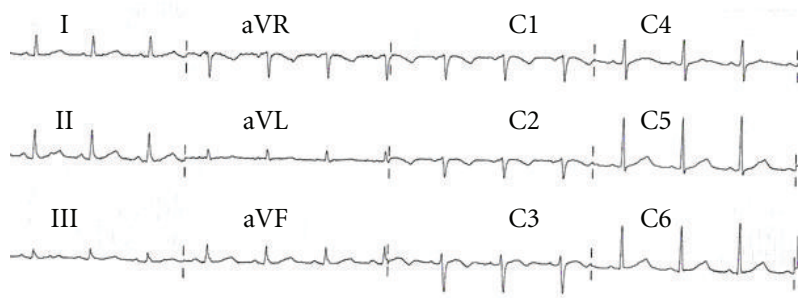

FIGURE 5: ECG one month later: sinus rhythm (82 bpm), QTc $478 \mathrm{~ms}$.

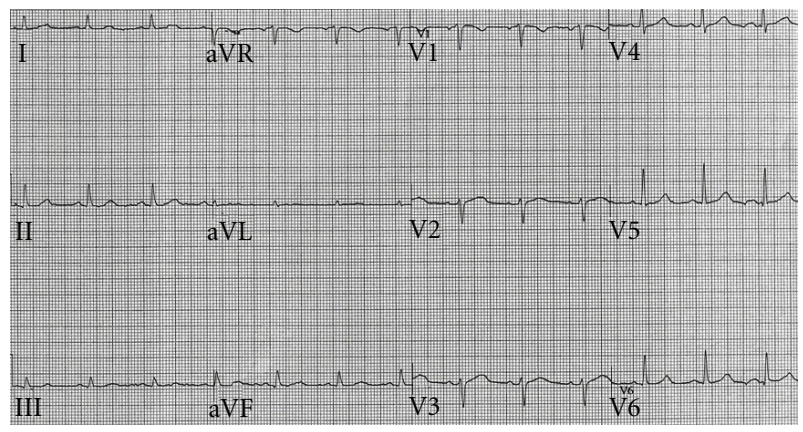

FIGURE 6: ECG six months later: normal sinus rhythm $73 \mathrm{bpm}$, QTc $400 \mathrm{~ms}$.

Myocardial ischemia should be always considered and ruled out, in case of TdPs and acquired long QT without bradycardia. As reported, coronary artery disease increases risk of fatal arrhythmic events in middle age patients with LQTS $[7,8]$. In all cases of acute presentation of life-threating arrhythmias, coronary artery disease should always be excluded with coronary angiogram or coronary CT. In fact, in our case after myocardial revascularization even in presence of QT interval prolongation no new arrhythmic events occurred; suggesting that acquired long QT interval and myocardial ischemia are indeed partners in the crime of recurring cardiac arrest.

\section{References}

[1] P. S. Mofrad, H. Rashid, and C. M. Tracy, "New-onset QT prolongation and torsades de pointes accompanied by left ventricular dysfunction secondary to acute pancreatitis," Pacing and Clinical Electrophysiology, vol. 26, no. 8, pp. 1765-1768, 2003.

[2] F. Kosar, F. Ates, I. Sahin, M. Karincaoglu, and B. Yildirim, "QT interval analysis in patients with chronic liver disease: a prospective study," Angiology, vol. 58, no. 2, pp. 218-224, 2007.

[3] F. Ates, F. Kosar, Y. Aksoy, B. Yildirim, I. Sahin, and F. Hilmioglu, "QT interval analysis in patients with acute biliary pancreatitis," Pancreas, vol. 31, no. 3, pp. 238-241, 2005.

[4] D. N. Kenigsberg, S. Khanal, M. Kowalski, and S. C. Krishnan, "Prolongation of the QTc interval is seen uniformly during early transmural ischemia," Journal of the American College of Cardiology, vol. 49, no. 12, pp. 1299-1305, 2007.

[5] M. Kawabata, K. Hirao, S. Takeshi et al., "Torsades de pointes related to transient marked QT prolongation following successful emergent percutaneous coronary intervention for acute coronary syndrome," Journal of Electrocardiology, vol. 41, no. 2, pp. 117-122, 2008.

[6] A. Y. Fung, C. R. Kerr, and T. K. Maybee, "QT prolongation and torsades de pointes: the sole manifestation of coronary artery disease," International Journal of Cardiology, vol. 7, no. 1, pp. 63-66, 1985.

[7] I. Goldenberg, A. J. Moss, D. R. Peterson et al., "Long-QT syndrome after age 40," Circulation, vol. 117, no. 17, pp. 21922201, 2008.

[8] E. Sze, A. J. Moss, I. Goldenberg et al., "Long QT syndrome in patients over 40 years of age: increased risk for LQTS-related cardiac events in patients with coronary disease," Annals of Noninvasive Electrocardiology, vol. 13, no. 4, pp. 327-331, 2008. 


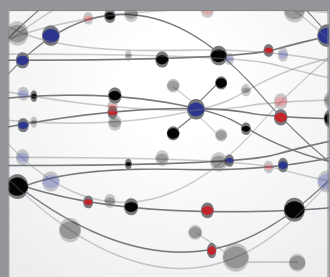

The Scientific World Journal
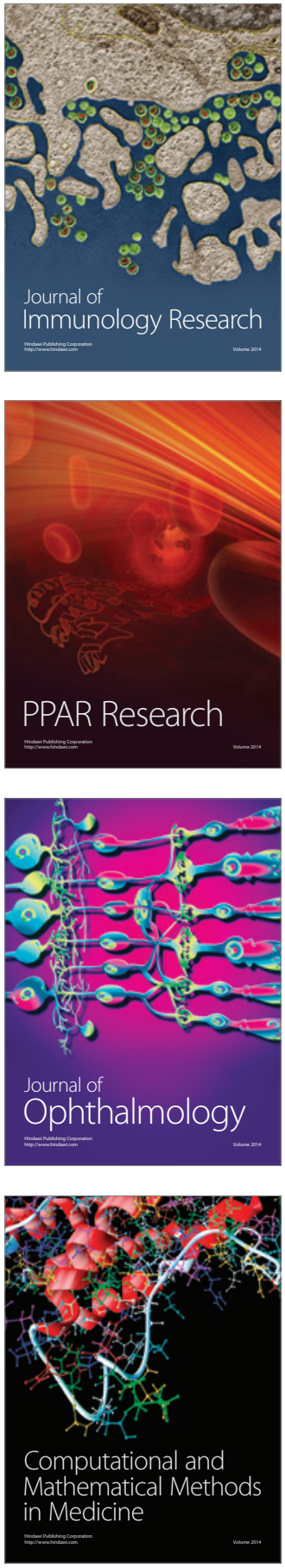

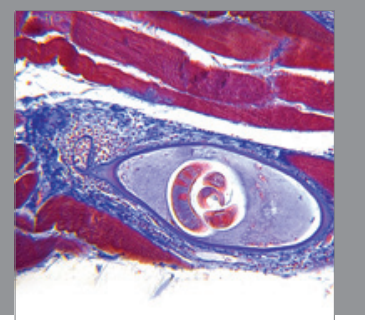

Gastroenterology

Research and Practice
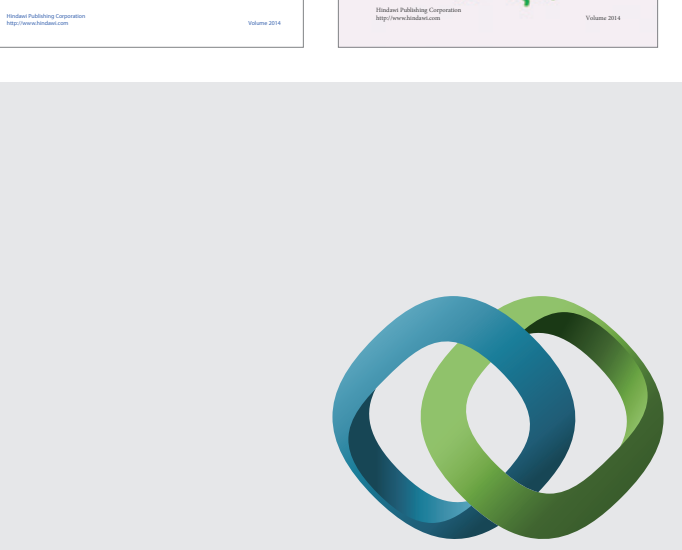

\section{Hindawi}

Submit your manuscripts at

http://www.hindawi.com
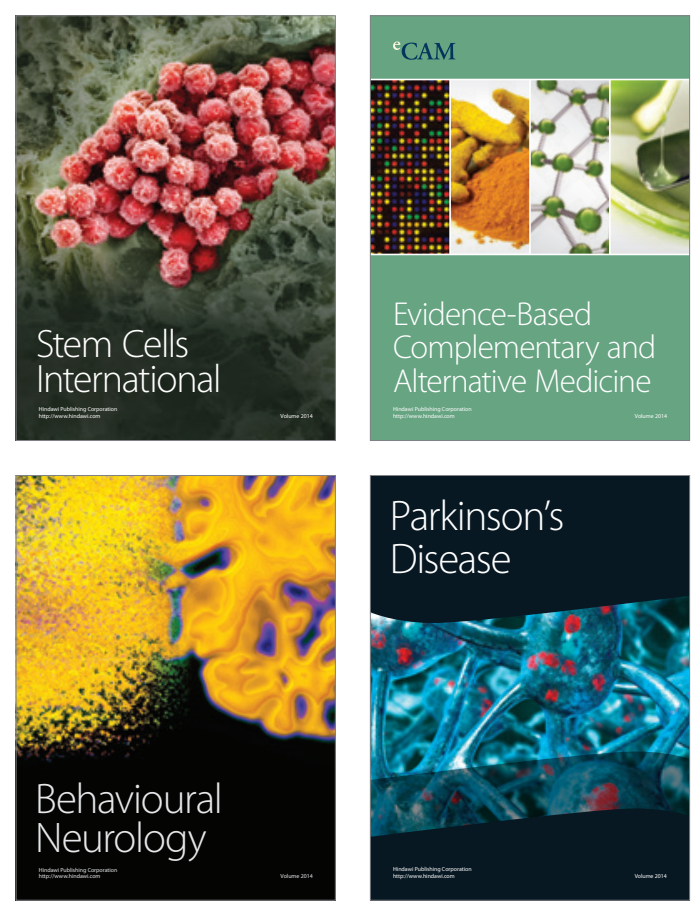

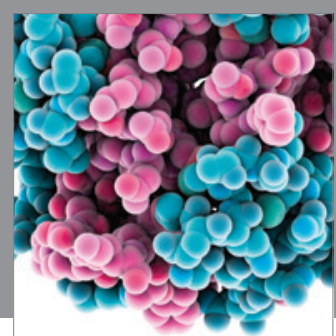

Journal of
Diabetes Research

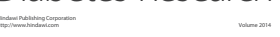

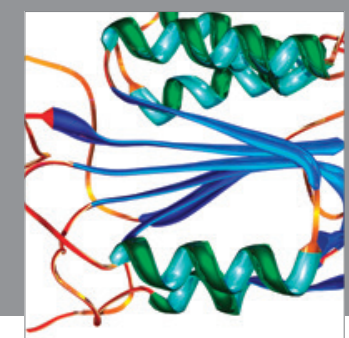

Disease Markers
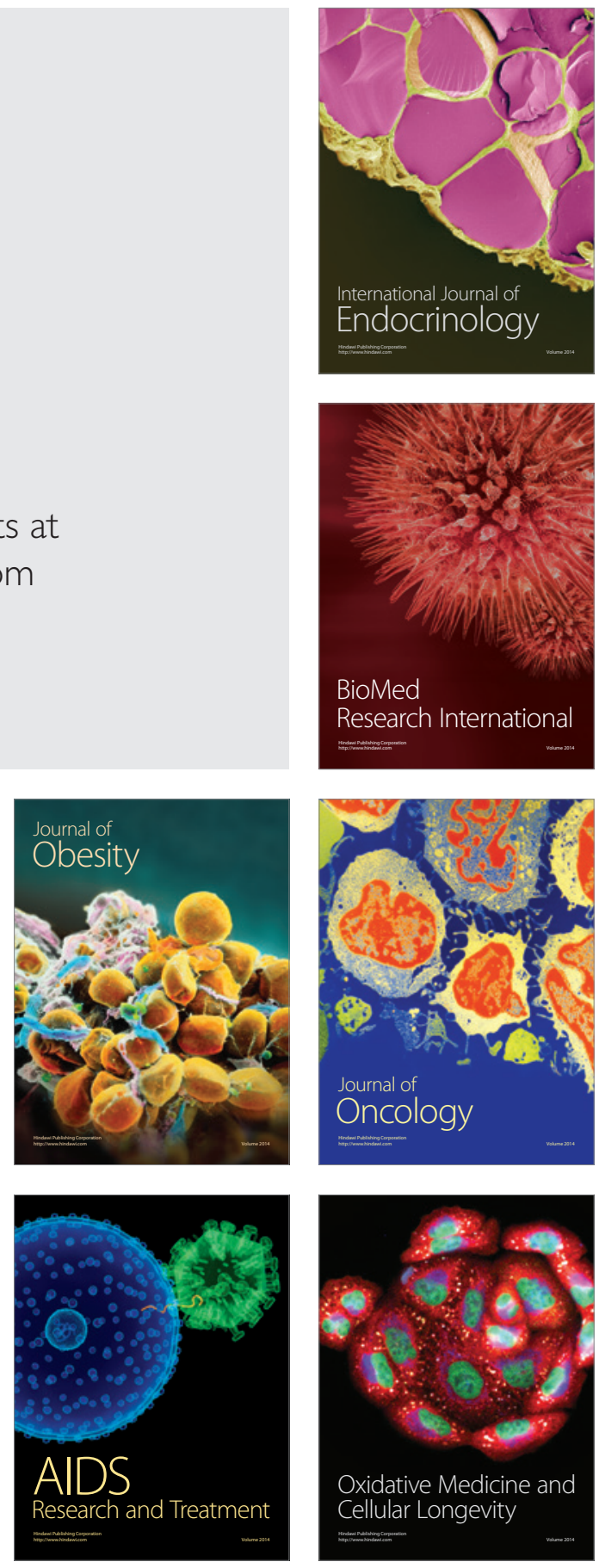